\title{
The contraction-proximal point algorithm with square-summable errors
}

\section{Changan Tian' and Fenghui Wang ${ }^{2 *}$}

"Correspondence:

wfenghui@gmail.com

${ }^{2}$ Department of Mathematics,

Luoyang Normal University,

Luoyang, 471022, P.R. China

Full list of author information is

available at the end of the article

\begin{abstract}
In this paper, we study the contraction-proximal point algorithm for approximating a zero of a maximal monotone mapping. The norm convergence of such an algorithm has been established under two new conditions. This extends a recent result obtained by Ceng, Wu and Yao to a more general case.
\end{abstract}

MSC: 47J20; 49J40; 65J15; 90C25

Keywords: maximal monotone operator; proximal point algorithm; firmly nonexpansive operator

\section{Introduction}

We consider the problem of finding $\hat{x} \in \mathcal{H}$ so that

$$
0 \in A \hat{x},
$$

where $\mathcal{H}$ is a Hilbert space and $A: \mathcal{H} \rightrightarrows \mathcal{H}$ is a given maximal monotone mapping. This problem is essential due to its various application in some concrete disciplines, including convex programming and variational inequalities. A classical way to solve such a problem is the proximal point algorithm (PPA) [1]. For any initial guess $x_{0} \in \mathcal{H}$, the PPA generates an iterative sequence as

$$
x_{n+1}=J_{c_{n}}\left(x_{n}+e_{n}\right) \text {, }
$$

where $J_{c_{n}}$ stands for the resolvent of $A$ and $\left(e_{n}\right)$ is the error sequence. In general, the following accuracy criterion on the error sequence:

$$
\left\|e_{n}\right\| \leq \epsilon_{n} \quad \text { with } \sum_{n=0}^{\infty} \epsilon_{n}<\infty
$$

is needed to ensure the convergence of PPA. In [1], Rockefeller also presented another accuracy criterion on the error sequence:

$$
\left\|e_{n}\right\| \leq \eta_{n}\left\|\tilde{x}_{n}-x_{n}\right\| \quad \text { with } \sum_{n=0}^{\infty} \eta_{n}<\infty,
$$

2013 Tian and Wang; licensee Springer. This is an Open Access article distributed under the terms of the Creative Commons Attribution License (http://creativecommons.org/licenses/by/2.0), which permits unrestricted use, distribution, and reproduction in any medium, provided the original work is properly cited. 
where

$$
\tilde{x}_{n}=J_{c_{n}}\left(x_{n}+e_{n}\right)
$$

This criterion was then improved by Han and $\mathrm{He}[2]$ as

$$
\left\|e_{n}\right\| \leq \eta_{n}\left\|\tilde{x}_{n}-x_{n}\right\| \quad \text { with } \sum_{n=0}^{\infty} \eta_{n}^{2}<\infty
$$

It is well known that the PPA does not necessarily converge strongly [3]. Then how to modify the PPA so that the strong convergence is guaranteed attracts serious attention of many researchers (see, e.g., [4-8]). In particular, one method for doing this has the following scheme:

$$
x_{n+1}=\lambda_{n} u+\left(1-\lambda_{n}\right) J_{c_{n}}\left(x_{n}+e_{n}\right),
$$

where $u \in \mathcal{H}$ is fixed and $\left(\lambda_{n}\right)$ is a real sequence. This algorithm, introduced independently by $\mathrm{Xu}$ [8] and Kamimura-Takahashi [5], is known as the contraction-proximal point algorithm (CPPA) [9], which is indeed a combination of Halpern's iteration and the PPA. There are various conditions that ensure the norm convergence of the CPPA with criterion (I) (cf. $[7,10-12])$ and the weakest one so far may be the following [13]:

(i) $c_{n} \geq c>0$;

(ii) $\lim _{n} \lambda_{n}=0, \sum_{n=0}^{\infty} \lambda_{n}=\infty$;

(iii) $\left\|e_{n}\right\| \leq \eta_{n}, \sum_{n=0}^{\infty} \eta_{n}<\infty$.

Let us now turn our attention to the CPPA under criterion (II). In this situation, Ceng, Wu and Yao [14] obtained the norm convergence under the following conditions:

(i) $\lim _{n} c_{n}=\infty$;

(ii) $\lim _{n} \lambda_{n}=0, \sum_{n=0}^{\infty} \lambda_{n}=\infty$;

(iii) $\left\|e_{n}\right\| \leq \eta_{n}\left\|\tilde{x}_{n}-x_{n}\right\|$ with $\sum_{n=0}^{\infty} \eta_{n}^{2}<\infty$.

In the hypothesis mentioned above, the sequence $\left(c_{n}\right)$ is assumed to tend to infinity, so it is natural to ask whether the norm convergence is still guaranteed for bounded $\left(c_{n}\right)$, especially for constant sequence. In the present paper, we shall answer this question affirmatively and relax condition $\lim _{n} c_{n}=\infty$ to a more general case:

$$
c_{n} \geq c>0,
$$

that is, we only need to assume the sequence $\left(c_{n}\right)$ is bounded below away from zero. The paper is organized as follows. In Section 2, we prove two useful lemmas that are very useful for proving the boundedness of the iteration. In Section 3, we establish norm convergence of the CPPA under two different conditions. As a result, we extend the corresponding result obtained in [14].

\section{Some lemmas}

We denote by ' $\rightarrow$ ' strong convergence, and ' $\rightarrow$ ' weak convergence. An operator $A: \mathcal{H} \rightrightarrows \mathcal{H}$ is called monotone if

$$
\langle u-v, x-y\rangle \geq 0,
$$


for any $u \in A x, v \in A y$; maximal monotone if its graph

$$
\mathcal{G}(A)=\{(x, y): x \in \mathcal{D}(A), y \in A x\}
$$

is not properly contained in the graph of any other monotone operator.

Let $C$ be a nonempty, closed and convex subset of $\mathcal{H}$. We use $P_{C}$ to denote the projection from $\mathcal{H}$ onto $C$; namely, for $x \in \mathcal{H}, P_{C} x$ is the unique point in $C$ with the property:

$$
\left\|x-P_{C} x\right\|=\min _{y \in C}\|x-y\| .
$$

It is well known that $P_{C} x$ is characterized by

$$
\left\langle x-P_{C} x, z-P_{C} x\right\rangle \leq 0, \quad \forall z \in C .
$$

A mapping $T: \mathcal{H} \rightarrow \mathcal{H}$ is called firmly nonexpansive if

$$
\|T x-T y\|^{2} \leq\|x-y\|^{2}-\|(I-T) x-(I-T) y\|^{2}
$$

for all $x, y \in \mathcal{H}$. Here and hereafter, we denote by

$$
J_{c}=(I+c A)^{-1}
$$

the resolvent of $A$, where $c>0$ and $I$ is the identity operator. The zero set of $A$ is denoted by $S:=\{x \in \mathcal{D}(A): 0 \in A x\}$. The resolvent operator has the following properties (see [15]).

Lemma 1 Let $A$ be a maximal monotone operator. Then

(i) $\mathcal{D}\left(J_{c}\right)=\mathcal{H}$;

(ii) $J_{c}$ is single-valued and firmly nonexpansive;

(iii) $\operatorname{Fix}\left(J_{c}\right)=S$, where $\operatorname{Fix}\left(J_{c}\right)$ denotes the fixed point set of $J_{c}$;

(iv) its graph $\mathcal{G}(A)$ is weak-to-strong closed in $\mathcal{H} \times \mathcal{H}$.

Since $J_{c}$ is firmly nonexpansive, this implies that

$$
\left\|J_{c} x-z\right\|^{2} \leq\|x-z\|^{2}-\left\|\left(I-J_{c}\right) x\right\|^{2}
$$

for all $x \in \mathcal{H}$ and all $z \in S$. In what follows, we present two lemmas that are very useful for proving the boundedness of the iterative sequence.

Lemma 2 Given $\beta>0$, let $\left(s_{n}\right)$ be a nonnegative real sequence satisfying

$$
s_{n+1} \leq\left(1-\lambda_{n}\right)\left(1+\epsilon_{n}\right) s_{n}+\lambda_{n} \beta,
$$

where $\left(\lambda_{n}\right) \subset(0,1)$ and $\left(\epsilon_{n}\right) \in \ell_{1}$ are real sequences. Then $\left(s_{n}\right)$ is bounded; more precisely,

$$
s_{n} \leq \max \left\{\beta, s_{0}\right\} \exp \left(\sum_{n=0}^{\infty} \epsilon_{n}\right)<\infty .
$$


Proof We first show the following estimates:

$$
s_{n+1} \leq \max \left\{\beta, s_{0}\right\} \prod_{k=0}^{n}\left(1+\epsilon_{k}\right), \quad \forall n \geq 0 .
$$

For $n=0$, we have

$$
\begin{aligned}
s_{1} & \leq\left(1-\lambda_{0}\right)\left(1+\epsilon_{0}\right) s_{0}+\lambda_{0} \beta \\
& \leq\left(1+\epsilon_{0}\right)\left[\left(1-\lambda_{0}\right) s_{0}+\lambda_{0} \beta\right] \\
& \leq \max \left\{\beta, s_{0}\right\}\left(1+\epsilon_{0}\right) .
\end{aligned}
$$

Assume $s_{n} \leq \max \left\{\beta, s_{0}\right\} \prod_{k=0}^{n-1}\left(1+\epsilon_{k}\right)$. Since $\max \left\{\beta, s_{0}\right\} \prod_{k=0}^{n-1}\left(1+\epsilon_{k}\right) \geq \beta$, we have

$$
\begin{aligned}
s_{n+1} & \leq\left(1+\epsilon_{n}\right)\left(1-\lambda_{n}\right) s_{n}+\lambda_{n} \beta \\
& \leq\left(1+\epsilon_{n}\right)\left[\left(1-\lambda_{n}\right) s_{n}+\lambda_{n} \beta\right] \\
& \leq\left(1+\epsilon_{n}\right) \max \left\{s_{0}, \beta\right\} \prod_{k=0}^{n-1}\left(1+\epsilon_{k}\right) \\
& =\max \left\{\beta, s_{0}\right\} \prod_{k=0}^{n}\left(1+\epsilon_{k}\right) .
\end{aligned}
$$

We thus verify inequality (5) by induction. Hence,

$$
\begin{aligned}
s_{n+1} & \leq \max \left\{\beta, s_{0}\right\} \prod_{k=0}^{n}\left(1+\epsilon_{k}\right) \\
& =\max \left\{\beta, s_{0}\right\} \exp \left(\sum_{k=0}^{n} \ln \left(1+\epsilon_{k}\right)\right) \\
& \leq \max \left\{\beta, s_{0}\right\} \exp \left(\sum_{k=0}^{\infty} \epsilon_{k}\right)<\infty,
\end{aligned}
$$

where the last inequality follows from the basic inequality: $\ln (1+x)<x$ for all $x>0$.

Lemma 3 Given $\beta>0$, let $\left(s_{n}\right)$ be a nonnegative real sequence satisfying

$$
s_{n+1} \leq\left(1-\lambda_{n}\right)\left(1+\epsilon_{n}\right) s_{n}+\lambda_{n} \beta
$$

where $\left(\lambda_{n}\right) \subset(0,1)$ and $\left(\epsilon_{n}\right) \subseteq[0, \infty)$ are real sequences. If $2 \epsilon_{n}\left(1-\lambda_{n}\right) \leq \lambda_{n}$, then $\left(s_{n}\right)$ is bounded; more precisely, $s_{n} \leq \max \left\{2 \beta, s_{0}\right\}<\infty$.

Proof Let $\tau_{n}=\lambda_{n}-\epsilon_{n}\left(1-\lambda_{n}\right)$. Then $\tau_{n} \in(0,1)$. It follows that

$$
\begin{aligned}
s_{n+1} & \leq\left(1+\epsilon_{n}\right)\left(1-\lambda_{n}\right) s_{n}+\lambda_{n} \beta \\
& =\left(1-\tau_{n}\right) s_{n}+\tau_{n}\left(\lambda_{n} \beta / \tau_{n}\right) \\
& \leq \max \left\{\lambda_{n} \beta / \tau_{n}, s_{n}\right\} .
\end{aligned}
$$


Since $2 \epsilon_{n}\left(1-\lambda_{n}\right) \leq \lambda_{n}$, we have

$$
\frac{\lambda_{n}}{\tau_{n}}=\frac{\lambda_{n}}{\lambda_{n}-\epsilon_{n}\left(1-\lambda_{n}\right)} \leq 2,
$$

which implies that

$$
s_{n+1} \leq \max \left\{2 \beta, s_{n}\right\} .
$$

By induction, we can show the result as desired.

We end this section by two useful lemmas. The first one is due to Maingé [16] and the second one is due to $\mathrm{Xu}[8]$.

Lemma 4 Let $\left(s_{n}\right)$ be a real sequence that does not decrease at infinity, in the sense that there exists a subsequence $\left(s_{n_{k}}\right)$ so that

$$
s_{n_{k}} \leq s_{n_{k}+1} \text { for all } k \geq 0 \text {. }
$$

For every $n>n_{0}$ define an integer sequence $(\tau(n))$ as

$$
\tau(n)=\max \left\{n_{0} \leq k \leq n: s_{k}<s_{k+1}\right\}
$$

Then $\tau(n) \rightarrow \infty$ as $n \rightarrow \infty$ and for all $n>n_{0}$

$$
\max \left(s_{\tau(n)}, s_{n}\right) \leq s_{\tau(n)+1}
$$

Lemma 5 Let $\left\{s_{n}\right\},\left\{c_{n}\right\} \subset \mathbb{R}^{+},\left\{\lambda_{n}\right\} \subset(0,1)$ and $\left\{b_{n}\right\} \subset \mathbb{R}$ be sequences such that

$$
s_{n+1} \leq\left(1-\lambda_{n}\right) s_{n}+b_{n}+c_{n} \text { for all } n \geq 0 \text {. }
$$

If $\lambda_{n} \rightarrow 0, \sum_{n=0}^{\infty} \lambda_{n}=\infty, \sum_{n=0}^{\infty} c_{n}<\infty$ and $\varlimsup_{n} b_{n} / \lambda_{n} \leq 0$, then $\lim _{n} s_{n}=0$.

\section{Convergence analysis}

In what follows, we assume that $A$ is a maximal monotone mapping and its zero set $S$ is nonempty. To establish the convergence, we need the following lemma, which is indeed proved in [2]. We present here a different proof that is mainly based on property of firmly nonexpansive mappings.

Lemma 6 Let $\eta \in(0,1 / 2), x, e \in \mathcal{H}$ and $\tilde{x}:=J_{c}(x+e)$. If $\|e\| \leq \eta\|x-\tilde{x}\|$, then

$$
\|\tilde{x}-z\|^{2} \leq\left(1+(2 \eta)^{2}\right)\|x-z\|^{2}-\frac{1}{2}\|\tilde{x}-x\|^{2}, \quad \forall z \in S .
$$

Proof Since $z \in \operatorname{Fix}\left(J_{c}\right)$, it follows from (4) that

$$
\begin{aligned}
\|\tilde{x}-z\|^{2} & \leq\|x+e-z\|^{2}-\left\|x+e-J_{c}(x+e)\right\|^{2} \\
& =\|(x-z)+e\|^{2}-\|(x-\tilde{x})+e\|^{2} \\
& =\|x-z\|^{2}+2\langle\tilde{x}-z, e\rangle-\|\tilde{x}-x\|^{2} .
\end{aligned}
$$


By using inequality $2\langle a, b\rangle \leq 2 \eta^{2}\|a\|^{2}+\|b\|^{2} / 2 \eta^{2}$, we have

$$
2\langle\tilde{x}-z, e\rangle \leq 2 \eta^{2}\|\tilde{x}-z\|^{2}+\frac{1}{2 \eta^{2}}\|e\|^{2} .
$$

Subsisting this into (8) and noting $\|e\| \leq \eta\|x-\tilde{x}\|$, we see that

$$
\|\tilde{x}-z\|^{2} \leq\|x-z\|^{2}+2 \eta^{2}\|\tilde{x}-z\|^{2}-\frac{1}{2}\|\tilde{x}-x\|^{2},
$$

from which it follows that

$$
\|\tilde{x}-z\|^{2} \leq\left(1+\frac{2 \eta^{2}}{1-2 \eta^{2}}\right)\|x-z\|^{2}-\frac{1}{2\left(1-2 \eta^{2}\right)}\|\tilde{x}-x\|^{2} .
$$

Consequently, the desired inequality (7) follows from the fact $\eta \in(0,1 / 2)$.

We now are ready to prove our main results.

Theorem 1 For any $x_{0} \in \mathcal{H}$, the sequence $\left(x_{n}\right)$ generated by

$$
\left[\begin{array}{l}
\tilde{x}_{n}=J_{c_{n}}\left(x_{n}+e_{n}\right), \\
x_{n+1}=\lambda_{n} u+\left(1-\lambda_{n}\right) \tilde{x}_{n},
\end{array}\right.
$$

converges strongly to $P_{S}(u)$, provided that

(i) $c_{n} \geq c>0$;

(ii) $\lim _{n} \lambda_{n}=0, \sum_{n=0}^{\infty} \lambda_{n}=\infty$;

(iii) $\left\|e_{n}\right\| \leq \eta_{n}\left\|\tilde{x}_{n}-x_{n}\right\|, \sum_{n=0}^{\infty} \eta_{n}^{2}<\infty$.

Proof Let $z=P_{S}(u)$. By our hypothesis, we may assume without loss of generality that $\eta_{n} \in(0,1 / 2)$. Then by Lemma 6 , we have

$$
\left\|\tilde{x}_{n}-z\right\|^{2} \leq\left(1+\epsilon_{n}\right)\left\|x_{n}-z\right\|^{2}-\frac{1}{2}\left\|\tilde{x}_{n}-x_{n}\right\|^{2},
$$

where $\epsilon_{n}:=\left(2 \eta_{n}\right)^{2}$ satisfying $\sum_{n=0}^{\infty} \epsilon_{n}<\infty$. It then follows from (9) that

$$
\begin{aligned}
\left\|x_{n+1}-z\right\|^{2} & =\left\|\left(1-\lambda_{n}\right)\left(\tilde{x}_{n}-z\right)^{2}+\lambda_{n}(u-z)\right\|^{2} \\
& \leq\left(1-\lambda_{n}\right)\left\|\tilde{x}_{n}-z\right\|^{2}+\lambda_{n}\|u-z\|^{2},
\end{aligned}
$$

which together with (10) yields

$$
\left\|x_{n+1}-z\right\|^{2} \leq\left(1-\lambda_{n}\right)\left(1+\epsilon_{n}\right)\left\|x_{n}-z\right\|^{2}+\lambda_{n}\|u-z\|^{2} .
$$

Applying Lemma 2 to the last inequality, we conclude that $\left(x_{n}\right)$ is bounded.

It follows from the subdifferential inequality that

$$
\begin{aligned}
\left\|x_{n+1}-z\right\|^{2} & =\left\|\left(1-\lambda_{n}\right)\left(\tilde{x}_{n}-z\right)+\lambda_{n}(u-z)\right\|^{2} \\
& \leq\left(1-\lambda_{n}\right)\left\|\tilde{x}_{n}-z\right\|^{2}+2 \lambda_{n}\left\langle u-z, x_{n+1}-z\right\rangle .
\end{aligned}
$$


Combining this with (10) yields

$$
\begin{aligned}
\left\|x_{n+1}-z\right\|^{2} \leq & \left(1-\lambda_{n}\right)\left\|x_{n}-z\right\|^{2}-\frac{1-\lambda_{n}}{2}\left\|\tilde{x}_{n}-x_{n}\right\|^{2} \\
& +2 \lambda_{n}\left\langle u-z, x_{n+1}-z\right\rangle+M \epsilon_{n},
\end{aligned}
$$

where $M>0$ is a sufficiently large number. Since $\left(\epsilon_{n}\right) \in \ell_{1}$, we assume that

$$
s:=\lim _{n \rightarrow \infty} \sum_{k=0}^{n} \epsilon_{k}<\infty
$$

and define $t_{n}:=s-\sum_{k=0}^{n-1} \epsilon_{k}$. Setting $s_{n}=\left\|x_{n}-z\right\|^{2}+M t_{n}$, we rewrite (11) as

$$
s_{n+1}-s_{n}+\lambda_{n}\left\|x_{n}-z\right\|^{2}+\frac{1-\lambda_{n}}{2}\left\|\tilde{x}_{n}-x_{n}\right\|^{2} \leq 2 \lambda_{n}\left\langle u-z, x_{n+1}-z\right\rangle .
$$

It is obvious that $s_{n} \rightarrow 0 \Leftrightarrow\left\|x_{n}-z\right\| \rightarrow 0$.

We next consider two possible cases on the sequence $\left(s_{n}\right)$.

Case 1. $\left(s_{n}\right)$ is eventually decreasing (i.e., there exists $N \geq 0$ such that $\left(s_{n}\right)$ is decreasing for $n \geq N)$. In this case, $\left(s_{n}\right)$ must be convergent, and from (12) it follows

$$
\frac{1-\lambda_{n}}{2}\left\|\tilde{x}_{n}-x_{n}\right\|^{2} \leq\left(s_{n}-s_{n+1}\right)+M \lambda_{n} \rightarrow 0,
$$

from which we have $\left\|\tilde{x}_{n}-x_{n}\right\| \rightarrow 0$. Extract a subsequence $\left(x_{n_{k}}\right)$ from $\left(x_{n}\right)$ so that $\left(x_{n_{k}}\right)$ converges weakly to $\hat{x}$ and

$$
\varlimsup_{n \rightarrow \infty}\left\langle u-z, x_{n+1}-z\right\rangle=\lim _{k \rightarrow \infty}\left\langle u-z, x_{n_{k}}-z\right\rangle .
$$

By noting the fact that $\tilde{x}_{n}=J_{n}\left(x_{n}+e_{n}\right)$, this implies

$$
0 \leftarrow \frac{x_{n_{k}}+e_{n_{k}}-\tilde{x}_{n_{k}}}{c_{n_{k}}} \in A\left(\tilde{x}_{n_{k}}\right)
$$

and $\tilde{x}_{n_{k}}=x_{n_{k}}+\left(\tilde{x}_{n_{k}}-x_{n_{k}}\right) \rightarrow \hat{x}$. Hence, the weak-to-strong closedness of $\mathcal{G}(A)$ implies $0 \in A(\hat{x})$, i.e., $\hat{x} \in S$. Consequently, we have

$$
\varlimsup_{n \rightarrow \infty}\left\langle u-z, x_{n+1}-z\right\rangle=\langle u-z, \hat{x}-z\rangle \leq 0,
$$

where the inequality follows from (3). Again it follows from (12) that

$$
\left\|x_{n+1}-z\right\|^{2} \leq\left(1-\lambda_{n}\right)\left\|x_{n}-z\right\|^{2}+2 \lambda_{n}\left\langle u-z, x_{n+1}-z\right\rangle+M \epsilon_{n} .
$$

By using Lemma 5 , we conclude that $\left\|x_{n}-z\right\| \rightarrow 0$.

Case 2. $\left(s_{n}\right)$ is not eventually decreasing. Hence, we can find a subsequence $\left(s_{n_{k}}\right)$ so that $s_{n_{k}} \leq s_{n_{k}+1}$ for all $k \geq 0$. In this case, we may define an integer sequence $(\tau(n))$ as in Lemma 4. Since $s_{\tau(n)} \leq s_{\tau(n)+1}$ for all $n>n_{0}$, it follows again from (12) that

$$
\frac{1-\lambda_{\tau(n)}}{2}\left\|\tilde{x}_{\tau(n)}-x_{\tau(n)}\right\|^{2} \leq M \lambda_{\tau(n)} \rightarrow 0,
$$


so that $\left\|\tilde{x}_{\tau(n)}-x_{\tau(n)}\right\| \rightarrow 0$ as $n \rightarrow \infty$. Analogously,

$$
\varlimsup_{n \rightarrow \infty}\left\langle u-z, x_{\tau(n)}-z\right\rangle \leq 0 .
$$

On the other hand, we deduce from (9) that

$$
\left\|x_{\tau(n)}-x_{\tau(n)+1}\right\| \leq \lambda_{\tau(n)}\left\|u-x_{\tau(n)}\right\|+\left\|\tilde{x}_{\tau(n)}-x_{\tau(n)}\right\| \rightarrow 0,
$$

which together with (13) gets

$$
\varlimsup_{n \rightarrow \infty}\left\langle u-z, x_{\tau(n)+1}-z\right\rangle \leq 0 .
$$

Noting $s_{\tau(n)+1}-s_{\tau(n)} \geq 0$ and dividing by $\lambda_{\tau(n)}$ in (12), we arrive at

$$
\left\|x_{\tau(n)}-z\right\|^{2} \leq 2\left\langle u-z, x_{\tau(n)+1}-z\right\rangle
$$

for all $n>n_{0}$, which together with (14) yields

$$
\varlimsup_{n \rightarrow \infty}\left\|x_{\tau(n)}-z\right\| \leq 0 .
$$

In view of (6), we have

$$
\left\|x_{n}-z\right\|^{2}+M t_{n} \leq\left\|x_{\tau(n)+1}-z\right\|^{2}+M t_{\tau(n)+1} .
$$

Since $\left\|x_{\tau(n)}-z\right\| \rightarrow 0$ and $\left\|x_{\tau(n)+1}-x_{\tau(n)}\right\| \rightarrow 0$ implies $\left\|x_{\tau(n)+1}-z\right\| \rightarrow 0$, this together with the fact $t_{n} \rightarrow 0$ immediately yields $x_{n} \rightarrow z$.

For criterion (I), Boikanyo and Morosanu [10] introduced a new condition:

$$
\left\|e_{n}\right\| \leq \eta_{n} \quad \text { with } \lim _{n \rightarrow \infty} \frac{\eta_{n}}{\lambda_{n}}=0
$$

to ensure the convergence of the CPPA. In the following theorem, we shall present a similar condition under the accuracy criterion (II).

Theorem 2 For any $x_{0} \in \mathcal{H}$, the sequence $\left(x_{n}\right)$ generated by

$$
\left[\begin{array}{l}
\tilde{x}_{n}=J_{c_{n}}\left(x_{n}+e_{n}\right), \\
x_{n+1}=\lambda_{n} u+\left(1-\lambda_{n}\right) \tilde{x}_{n},
\end{array}\right.
$$

converges strongly to $P_{S}(u)$, provided that

(i) $c_{n} \geq c>0$

(ii) $\lim _{n} \lambda_{n}=0, \sum_{n=0}^{\infty} \lambda_{n}=\infty$;

(iii) $\left\|e_{n}\right\| \leq \eta_{n}\left\|\tilde{x}_{n}-x_{n}\right\|, \lim _{n} \eta_{n}^{2} / \lambda_{n}=0$.

Proof Let $z=P_{S}(u)$. Similarly, we have

$$
\left\|x_{n+1}-z\right\|^{2} \leq\left(1-\lambda_{n}\right)\left(1+\epsilon_{n}\right)\left\|x_{n}-z\right\|^{2}+\lambda_{n}\|u-z\|^{2},
$$


where $\epsilon_{n}:=\left(2 \eta_{n}\right)^{2}$ satisfying $\epsilon_{n} / \lambda_{n} \rightarrow 0$, so we assume without loss of generality that $2 \epsilon_{n}\left(1-\lambda_{n}\right) \leq \lambda_{n}$. Applying Lemma 3 , we conclude that $\left(x_{n}\right)$ is bounded.

From inequality (11), we also obtain

$$
s_{n+1}-s_{n}+\lambda_{n} s_{n}+\frac{1-\lambda_{n}}{2}\left\|\tilde{x}_{n}-x_{n}\right\|^{2} \leq 2 \lambda_{n}\left\langle u-z, x_{n+1}-z\right\rangle+s_{n} \epsilon_{n}
$$

where we define $s_{n}:=\left\|x_{n}-z\right\|^{2}$.

To show $s_{n} \rightarrow 0$, we consider two possible cases for $\left(s_{n}\right)$.

Case 1. $\left(s_{n}\right)$ is eventually decreasing (i.e., there exists $N \geq 0$ such that $\left(s_{n}\right)$ is decreasing for $n \geq N)$. In this case, $\left(s_{n}\right)$ must be convergent, and from (12) it follows

$$
\frac{1-\lambda_{n}}{2}\left\|\tilde{x}_{n}-x_{n}\right\|^{2} \leq\left(s_{n}-s_{n+1}\right)+M\left(\epsilon_{n}+\lambda_{n}\right) \rightarrow 0,
$$

where $M>0$ a sufficiently large number. Analogous to the previous theorem,

$$
\varlimsup_{n \rightarrow \infty}\left\langle u-z, x_{n+1}-z\right\rangle \leq 0 .
$$

Rearranging terms in (17) yields that

$$
s_{n+1} \leq\left(1-\lambda_{n}\right) s_{n}+\lambda_{n} M\left(2\left\langle u-z, x_{n+1}-z\right\rangle+\epsilon_{n} / \lambda_{n}\right) .
$$

We note that by our hypothesis $\epsilon_{n} / \lambda_{n}$ goes to zero, and thus apply Lemma 5 to the previous inequality to conclude that $s_{n} \rightarrow 0$.

Case $2 .\left(s_{n}\right)$ is not eventually decreasing. In this case, we may define an integer sequence $(\tau(n))$ as in Lemma 4. Since $s_{\tau(n)} \leq s_{\tau(n)+1}$ for all $n>n_{0}$, it follows again from (17) that

$$
\frac{1-\lambda_{\tau(n)}}{2}\left\|\tilde{x}_{\tau(n)}-x_{\tau(n)}\right\|^{2} \leq M\left(\lambda_{\tau(n)}+\epsilon_{\tau(n)}\right) \rightarrow 0,
$$

so that $\left\|\tilde{x}_{\tau(n)}-x_{\tau(n)}\right\| \rightarrow 0$, and furthermore $\left\|x_{\tau(n)}-x_{\tau(n)+1}\right\| \rightarrow 0$. Analogously,

$$
\varlimsup_{n \rightarrow \infty}\left\langle u-z, x_{\tau(n)+1}-z\right\rangle \leq 0 .
$$

It follows from (17) that for all $n>n_{0}$

$$
s_{\tau(n)} \leq 2\left\langle u-z, x_{\tau(n)+1}-z\right\rangle+\frac{M \epsilon_{\tau(n)}}{\lambda_{\tau(n)}} .
$$

By combining the last two inequalities, we have

$$
\varlimsup_{n \rightarrow \infty} s_{\tau(n)} \leq 0
$$

from which we arrive at

$$
\begin{aligned}
\sqrt{s_{\tau(n)+1}} & =\left\|\left(x_{\tau(n)}-z\right)-\left(x_{\tau(n)}-x_{\tau(n)+1}\right)\right\| \\
& \leq \sqrt{s_{\tau(n)}}+\left\|x_{\tau(n)}-x_{\tau(n)+1}\right\| \rightarrow 0 .
\end{aligned}
$$

Consequently, $s_{n} \rightarrow 0$ follows from (6) immediately. 


\section{Competing interests}

The authors declare that they have no competing interests.

\section{Authors' contributions}

Both authors contributed equally and significantly to writing this manuscript. Both authors read and approved the manuscript.

\section{Author details}

${ }^{1}$ Department of Mathematics, Henan Normal University, Xinxiang, 453007, P.R. China. ${ }^{2}$ Department of Mathematics, Luoyang Normal University, Luoyang, 471022, P.R. China.

\section{Acknowledgements}

The authors thank the referees for their useful comments and suggestions. This work is supported by the National Natural Science Foundation of China, Tianyuan Foundation (11226227), the Basic Science and Technological Frontier Project of Henan (122300410268, 122300410375).

Received: 7 September 2012 Accepted: 26 March 2013 Published: 11 April 2013

\section{References}

1. Rockafellar, RT: Monotone operators and the proximal point algorithm. SIAM J. Control Optim. 14, $877-898$ (1976)

2. Han, D, He, BS: A new accuracy criterion for approximate proximal point algorithms. J. Math. Anal. Appl. 263, 343-354 (2001)

3. Güler, O: On the convergence of the proximal point algorithm for convex optimization. SIAM J. Control Optim. 29 403-419 (1991)

4. Bauschke, $\mathrm{HH}$, Combettes, PL: A weak-to-strong convergence principle for Fejér-monotone methods in Hilbert spaces. Math. Oper. Res. 26, 248-264 (2001)

5. Kamimura, S, Takahashi, W: Approximating solutions of maximal monotone operators in Hilbert spaces. J. Approx Theory 106, 226-240 (2000)

6. Solodov, MV, Svaiter, BF: Forcing strong convergence of proximal point iterations in a Hilbert space. Math. Program. 87, 189-202 (2000)

7. Wang, F: A note on the regularized proximal point algorithm. J. Glob. Optim. 50, 531-535 (2011)

8. Xu, HK: Iterative algorithms for nonlinear operators. J. Lond. Math. Soc. 66, 240-256 (2002)

9. Marino, G, Xu, HK: Convergence of generalized proximal point algorithm. Commun. Pure Appl. Anal. 3, 791-808 (2004)

10. Boikanyo, OA, Morosanu, G: A proximal point algorithm converging strongly for general errors. Optim. Lett. 4 635-641 (2010)

11. Boikanyo, OA, Morosanu, G: Four parameter proximal point algorithms. Nonlinear Anal. 74, 544-555 (2011)

12. Xu, HK: A regularization method for the proximal point algorithm. J. Glob. Optim. 36, 115-125 (2006)

13. Wang, F, Cui, H: On the contraction-proximal point algorithms with multi-parameters. J. Glob. Optim. 54, 485-491 (2012)

14. Ceng, LC, Wu, SY, Yao, JC: New accuracy criteria for modified approximate proximal point algorithms in Hilbert space. Taiwan. J. Math. 12, 1691-1705 (2008)

15. Bauschke, HH, Combettes, PL: Convex Analysis and Monotone Operator Theory in Hilbert Spaces. Springer, New York (2011)

16. Maingé, PE: Strong convergence of projected subgradient methods for nonsmooth and nonstrictly convex minimization. Set-Valued Anal. 16, 899-912 (2008)

doi:10.1186/1687-1812-2013-93

Cite this article as: Tian and Wang: The contraction-proximal point algorithm with square-summable errors. Fixed Point Theory and Applications 2013 2013:93.

\section{Submit your manuscript to a SpringerOpen ${ }^{\circ}$ journal and benefit from:}

- Convenient online submission

- Rigorous peer review

- Immediate publication on acceptance

- Open access: articles freely available online

- High visibility within the field

- Retaining the copyright to your article 